\title{
Editorial
}

\section{Targeting the Laminated Layer of Echinococcus multilocularis as a Potential Therapeutic Strategy}

\author{
Richard D. Cummings ${ }^{\text {a }}$ Stephan von Gunten ${ }^{b}$ \\ aDepartment of Surgery, Beth Israel Deaconess Medical Center, Harvard Medical School, Boston, MA, USA; ${ }^{\mathrm{b}}$ Institute \\ of Pharmacology, University of Bern, Bern, Switzerland
}

Alveolar echinococcosis (AE) caused by Echinococcus multilocularis is among the most lethal helminthic diseases, but current therapeutic strategies are suboptimal due to poor parasitocidal efficacy and toxicity $[1,2]$. Upon accidental ingestion of parasite eggs originating from feces of carnivore hosts (e.g., fox and dogs), eggs hatch and subsequently larval oncospheres penetrate the intestinal lining. Following migration primarily to the liver, they develop into vesiculated cysts, which are thickwall protected and continuously proliferate by external budding. Larvae can secondarily also develop in extrahepatic tissues (such as lungs and brain), resulting in a complicated course of disease, particularly in immunocompromised individuals [3], or patients remaining untreated. The E. multilocularis vesicles/microcysts are protected from physiological and immunological host responses by a carbohydrate-rich laminated layer (LL), mainly composed of evolutionary conserved core- 1 and 2 mucin-type $O$-glycans, and an elongated core 2 structure with a terminal $\alpha$-linked galactose $(\alpha-\mathrm{Gal})$ cap $[4,5]$.

In this issue of Pharmacology, Wang et al. [6] demonstrate that enzymatic digestion of the LL of E. multilocularis may offer a novel therapeutic approach. Treatment of cultured E. multilocularis metacestodes (clone H95) with either $\alpha 1-3,4,6$ or $\beta 1-3$ galactosidases both resulted in the collapse of vesicles starting after 1 day and the destruction of intravesicular structures 5 days posttreatment. Intraperitoneal injection of untreated parasite vesicles in mice resulted, as expected, in multivesiculated tumor-like structures. By contrast only small calcified lesions and significantly reduced parasite weight was observed upon pre-treatment of the vesicles with either $\alpha$ - or $\beta$-galactosidase, which indicates the importance of the LL for parasite survival. Although treatment by both enzymes resulted in an "abortive" course of infection, differences in the subsequent immune response were observed, including lower numbers of intrahepatic IL-10+ $\mathrm{CD} 4^{+} \mathrm{T}$ cells upon treatment with $\beta$-galactosidase.

The mechanistic roles of glycans with in the LL in parasite survival are uncertain. In humans, $\alpha-G a l$ structures, which are frequently found in pathogens, induce anti- $\alpha-$ Gal antibodies and are common in the serum of healthy individuals [7]. Whether the remarkable reduction of anti- $\alpha-G a l$ antibodies in immunodeficient patients [8], or in some healthy individuals [9], may influence the incidence or course of AE remains to be studied. While $\beta$-galactosidase treatment, as used in the experiments by Wang et al. [6], might digest the core structures of the $E$. karger@karger.com www.karger.com/pha

(C) 2021 S. Karger AG, Basel

Karger"
Stephan von Gunten

Institute of Pharmacology INO-F, Inselspital, University of Bern $\mathrm{CH}-3010$ Bern (Switzerland)

stephan.vongunten@pki.unibe.ch 
multilocularis LL, the removal of the $\alpha$-Gal cap appeared to be similarly effective, yet resulted in an altered immune response. While the destruction of the LL may help to overcome a major barrier of resistance, future studies will be required to investigate the subsequent host-pathogen responses and interactions, which may necessitate additional and personalized therapeutic approaches $[3,10]$, in light of the importance of the hosts' genotype in AE susceptibility and resistance $[2,11]$.

\section{Conflict of Interest Statement}

The author declares no conflicts of interest.

\section{Funding Sources}

The authors did not receive any funding.

\section{Author Contributions}

S.V.G. and R.D.G. wrote the manuscript.

\section{References}

1 Hemphill A, Stadelmann B, Rufener R, Spiliotis M, Boubaker G, Müller J, et al. Treatment of echinococcosis: albendazole and mebendazole-what else? Parasite. 2014;21:70.

2 Wen H, Vuitton L, Tuxun T, Li J, Vuitton DA, Zhang W, et al. Echinococcosis: advances in the 21st century. Clin Microbiol Rev. 2019 Feb 13;32(2):e00075-18.

3 Meinel TR, Gottstein B, Geib V, Keel MJ, Biral R, Mohaupt M, et al. Vertebral alveolar echinococcosis: a case report, systematic analysis, and review of the literature. Lancet Infect Dis. 2018 Mar;18(3):e87-98.

4 del Puerto L, Rovetta R, Navatta M, Fontana C, Lin G, Moyna G, et al. Negligible elongation of mucin glycans with Gal $\beta 1-3$ units distinguishes the laminated layer of Echinococcus multilocularis from that of Echinococcus granulosus. Int J Parasitol. 2016;46(5-6):311.
5 Hülsmeier AJ, Gehrig PM, Geyer R, Sack R, Gottstein B, Deplazes P, et al. A major Echinococcus multilocularis antigen is a mucintype glycoprotein. J Biol Chem. 2002;277(8): 5742 .

6 Wang J, von Gunten S, Beldi G, Grandgirard D, Leib SL, Gottstein B. Digest the sugar, kill the parasite: a new experimental concept in treating alveolar echinococcosis. Pharmacology.2020.https://doi.org/10.1159/000509355.

7 Schneider C, Smith DF, Cummings RD, Boligan KF, Hamilton RG, Bochner BS, et al. The human IgG anti-carbohydrate repertoire exhibits a universal architecture and contains specificity for microbial attachment sites. Sci Transl Med. 2015;7(269):269ra1.
8 Jandus $\mathrm{P}$, Frias Boligan K, Smith DF, de Graauw E, Grimbacher B, Jandus C, et al. The architecture of the IgG anti-carbohydrate repertoire in primary antibody deficiencies (PADs). Blood. 2019 Sep;134(22):1941-50.

9 Luetscher RND, McKitrick TR, Gao C, Mehta AY, McQuillan AM, Kardish R, et al. Unique repertoire of anti-carbohydrate antibodies in individual human serum. Sci Rep. 2020 Sep 22;10(1):15436.

10 von Gunten $S$. The future of pharmacology: towards more personalized pharmacotherapy and reverse translational research. Pharmacology. 2020;105(1-2):1-2.

11 Wang J, Gottstein B. Immunoregulation in larval Echinococcus multilocularis infection. Parasite Immunol. 2016;38(3):182. 